\title{
Hyperuricaemia in cyclosporin-treated patients: a GFR-related effect
}

\author{
R. M. Zürcher, H. A. Bock and G. Thiel \\ Division of Nephrology, Department of Medicine, University Hospital Basel, Switzerland
}

\begin{abstract}
Background. Hyperuricaemia is a well known sideeffect of cyclosporin A (CsA) treatment. The pathogenic mechanisms, however, remain controversial. There is no convincing evidence that hyperuricaemia is due to CsA-induced, impaired tubular handling of uric acid. The impact of diminished GFR in this
\end{abstract} particular context has never been investigated.

Methods. We prospectively studied plasma uric acid, inulin clearances, and fractional clearances of uric acid in two groups of CsA-treated patients (bone-marrow transplant patients, $n=50$; renal transplant patients, $n=32$ ), and one healthy control group without CsA (living related kidney donors, $n=28$ ). Bone-marrow transplant patients were examined before transplantation and $6,12,18,24$ months after transplantation, renal transplant patients 1 year after transplantation, and living related kidney donors before and 1 year after unilateral nephrectomy.

Results. After 1 year of CsA treatment, hyperuricaemia was found in $36 \%$ of bone-marrow transplant patients and in $53 \%$ of renal transplant patients. Thirty per cent of living related kidney donors were borderline hyperuricaemic I year after unilateral nephrectomy. The fractional clearance of uric acid, measured serially in bone-marrow transplant patients did not change significantly over time; it was, however, slightly higher during CsA treatment than after CsA withdrawal. Moreover, the bone-marrow transplant patients' fractional clearance of uric acid was not statistically different from the renal transplant patients' and the living related kidney donors' (values 1 year after transplantation/unilateral nephrectomy: bone-marrow transplant patients, $15.3 \pm 2.3 \%$; renal transplant patients, $11.9 \pm 0.9 \%$; living related kidney donors, $11.1 \pm 0.8 \%$ ). The GFR at 1 year, measured by inulin clearance, was identical in the CsA-treated groups and slightly higher in the living related kidney donors (bone-marrow transplant patients, $51 \pm 6 \mathrm{ml} / \mathrm{min}$ per $1.73 \mathrm{~m}^{2}$; renal transplant patients, $49 \pm 3 \mathrm{ml} / \mathrm{min}$ per $1.73 \mathrm{~m}^{2}$; living related kidney donors, $61 \pm 2 \mathrm{ml} / \mathrm{min}$ per $1.73 \mathrm{~m}^{2}$ ).

Conclusion. There is no evidence for impaired tubular handling of uric acid, induced by a CsA-specific tubulo-

Correspondence and offprint requests to: Regula Zurcher MD, Clinique de Médecine II, Hópital Cantonal Universitaire, rue Micheli-du-Crest 24, CH-1211 Genéve 14, Switzerland. toxic effect. Hyperuricaemia in CsA-treated transplant patients can therefore be attributed to the cyclosporinassociated decrease of GFR.

Key words: cyclosporin; bone-marrow transplantation; glomerular filtration rate; organ donors; renal transplantation; uric acid

\section{Introduction}

CsA-associated hyperuricaemia was described for the first time in 1983 [1]. Since then, several authors have confirmed this finding and have speculated about its pathogenesis, attributing hyperuricaemia to diminished renal function, diuretic use, or impaired tubular handling of uric acid [2-16]. The hypothesis of decreased fractional clearance of uric acid during CsA administration has been tested in its relationship with creatinine clearance $[4,5]$ and, except in one small study [6], has never been tested in relation to GFR itself, measured by classical inulin clearance. Diuretic use is a confounding variable in most studies. Furthermore, the fractional clearance of uric acid in CsA-treated patients has always been compared to azathioprinetreated patients and has never been formally compared to a healthy control group with comparable renal function but without immunosuppressive medication.

In order to define better the contribution of diminished GFR and tubular dysfunction respectively to hyperuricaemia, we conducted a prospective study, investigating inulin clearances and fractional clearances of uric acid in CsA-treated patients without (bonemarrow transplant patients) and with (renal transplant patients) underlying renal disease, as well as in a healthy control group without CsA and without kidney disease (living related kidney donors).

\section{Subjects and methods}

\section{Selection and treatment of patients}

Two groups of CsA-treated patients and one control group were studied: (a) a total of 50 consecutive patients who received CsA for allogenic bone marrow transplantation at 
the haematological unit of our hospital between 1988 and 1991 (CsA-group without underlying renal disease), (b) 32 consecutive CsA-treated patients who received a renal allograft from a living related kidney donor between 1988 and 1992 (group with underlying renal disease), and (c) 28 living related kidney donors (control group without underlying disease and without any immunosuppression) were entered in the study after having given written informed consent.

Investigations were performed as follows: plasma uric acid, inulin and creatinine clearances, and fractional clearance of uric acid before and $6,12,18$ and 24 months after bonemarrow transplantation (regardless of whether or not CsA was discontinued), 1 year after renal transplantation, and before and 1 year after unilateral nephrectomy respectively.

Exclusion criteria: bone-marrow transplant patients who could not be followed for up to at least 12 months (because of death, relapse of leukaemia, severe infections, noncompliance), were excluded from analysis $(n=23)$. Moreover, single investigations were excluded when patients received drugs that alter the synthesis or excretion of uric acid. Concomitant drugs: maintenance immunosuppression consisted of CsA alone or in combination with low-dose prednisone in bone-marrow transplant patients (mean CsA dose $280 \mathrm{mg} /$ day) and of CsA monotherapy $(n=21)$, CsA-azathioprine $(n=3), \quad$ CsA-azathioprine-prednisone $(n=7)$ and CsA-prednisone $(n=1)$ in the renal transplant group (mean CsA dose $300 \mathrm{mg}$ ). Diuretics were used by none of the bone-marrow transplant patients after transplantation. In the renal transplant group 21 of 32 patients received one or several antihypertensive drugs, only five of them, however, in combination with a diuretic (furosemide). None of the living related kidney donors was on diuretic therapy. At timepoint 0 of bone-marrow transplantation, most patients were on a multidrug regimen, including antimicrobial and antiviral drugs.

According to normal range values of the laboratory in our hospital, hyperuricaemia was considered to be present when the plasma uric acid level was above $490 \mu \mathrm{mol} / \mathrm{l}$ in men and above $360 \mu \mathrm{mol} / 1$ in women.

\section{Clearance techniques}

GFR was measured by standard inulin-clearance techniques: patients were admitted to our nephrology ward and well hydrated on the eve of the examination day ( 1 litre of water orally for all but the bone-marrow-transplant patients before transplantation, who usually received 3 litres of i.v. saline in glucose per $24 \mathrm{~h}$ ). The patients also received their evening meal and breakfast, each standardized in protein and salt content. CsA as well as other drugs were withheld until the end of the clearance period on the study day.

On the morning of the examination day, the patients were further hydrated, first with i.v. glucose $5 \%, 5 \mathrm{ml} / \mathrm{kg}$ body weight over $30 \mathrm{~min}$. The infusion was then changed to inulin in $0.9 \%$ saline. The dose of inulin was calculated to provide an inulin blood concentration of $30 \mathrm{mg} / 100 \mathrm{ml}$. After the loading dose was infused over $10-15 \mathrm{~min}$, a maintenance dose of inulin was delivered by a constant infusion pump. Sixty minutes were allowed for equilibration. Then four timed urine collections were obtained by spontaneous voiding at 40 -min intervals. After voiding, the patients drank $250 \mathrm{ml}$ of water to allow urine flow rates $>5 \mathrm{ml} / \mathrm{min}$. Blood samples were drawn midway into each collection period. Clearance values for inulin, creatinine and fractional clearance of uric acid were calculated for each period. The results given below are the mean \pm SEM of the four clearance periods. All clearance values are expressed per $1.73 \mathrm{~m}^{2}$ body surface area.

\section{Laboratory methods}

Uric acid concentrations in plasma and urine were measured using a uricase method (Hitachi 737, Boehringer Mannheim), plasma and urine creatinine concentrations by a creatininase assay (Wako Chemicals $\mathrm{GmbH}$, Neuss; Hitachi 737, Boehringer Mannheim). Inulin concentrations were measured by perchlorate hydrolysis.

\section{Statistical analysis}

Statistical evaluation was performed using one-way ANOVA for unpaired tests and paired $t$ tests for paired analysis. Correlations were evaluated using linear least squares regression; $P<0.05$ was considered significant and two-sided tests were used throughout. Data are given as mean \pm SEM.

\section{Results}

\section{Plasma uric acid}

Plasma uric acid levels of bone-marrow transplant patients were comparable to those of renal transplant patients under CsA ( $P$ NS; Table 1$)$. Plasma uric acid rose significantly during CsA therapy in bone-marrow transplant patients (paired $t$ test; $n=14 ; P<0.05$ ) and decreased to normal levels after CsA was stopped (Figure 1). This phenomenon was similar in males and females. Living related kidney donors experienced a significant rise in plasma uric acid after nephrectomy (paired $t$ test; $P<0.001$ ), 9 of 28 becoming borderline hyperuricaemic.

\section{Fractional clearance of uric acid and inulin clearance}

In bone-marrow transplant patients the fractional clearance of uric acid remained stable over the whole range of GFR examined, i.e. between an inulin clearance of 106 (before bone-marrow transplantation) to $45 \mathrm{ml} / \mathrm{min}$ per $1.73 \mathrm{~m}^{2}$ ( 24 months after bone-marrow transplantation), regardless of whether CsA was used or not (Table $2 \mathrm{a}, \mathrm{b}$ ). There were no statistically significant changes between values obtained before CsA was introduced, those obtained under CsA therapy, and those obtained after withdrawal of the drug. If anything, there was a tendency towards higher values during CsA therapy compared to those after discontinuing it. The renal transplant patients receiving CsA therapy had values for the fractional clearance of uric acid and inulin clearances comparable to those of the bone-marrow transplant patients (Table $2 a, b$ ). The fractional clearance of uric acid of the living related kidney donors was somewhat lower but not statistically different from that of the two patient populations under CsA (Table 2a).

Likewise, there were no differences in paired tests of 
Table 1. Plasma uric acid (P-UA)

\begin{tabular}{|c|c|c|c|c|c|c|c|c|}
\hline & \multicolumn{4}{|c|}{ P-UA \& $(\mu \mathrm{mol} / \mathrm{l})$} & \multicolumn{4}{|c|}{ P.UA oै ( $\mu \mathrm{mol} / 1)$} \\
\hline & $n$ & $\operatorname{Cs} A+$ & $n$ & $\mathrm{CsA}-$ & $n$ & $\operatorname{cs} \mathrm{A}+$ & $n$ & $\mathrm{Cs} \mathrm{A}-$ \\
\hline BMT 0 & & & $8^{*}$ & $232 \pm 19$ & & & $13^{*}$ & $303 \pm 23$ \\
\hline BMT $6 \mathrm{~m}$ & 9 & $366+30$ & & & 12 & $449 \pm 34$ & & \\
\hline BMT $12 m$ & 7 & $411 \pm 53$ & 4 & $271 \pm 21$ & 6 & $477 \pm 38$ & 10 & $351 \pm 18$ \\
\hline BMT $18 \mathrm{~m}$ & 6 & $367 \pm 23$ & 4 & $255 \pm 39$ & 6 & $502 \pm 23$ & 6 & $381 \pm 18$ \\
\hline BMT 24m & 3 & $409+44$ & 4 & $259+46$ & 2 & $490 \pm 49$ & 14 & $351 \pm 19$ \\
\hline LRKDO & & & 16 & $290+16$ & & & 12 & $407 \pm 17$ \\
\hline LRKD ly & & & 16 & $323+16$ & & & 12 & $451 \pm 14$ \\
\hline RTP 1y & 13 & $404 \pm 24$ & & & 19 & $456 \pm 19$ & & \\
\hline
\end{tabular}

Data are given as mean $t S E M$.

$B M T$, Bone-marrow transplant patients $(0=$ before; $6,12,18,24$ months after $t x)$.

LRKD, Living related kidney donors $\left(0=\right.$ before, $l_{y}=1$ year after unilateral $\left.n x\right)$.

RTP, Renal transplant patients $(1 y=1$ year after $t x)$.

* Patients on allopurinol excluded.

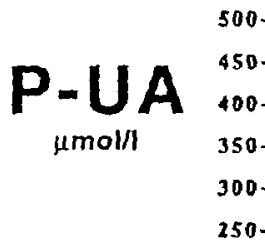

fCUA

$\%$
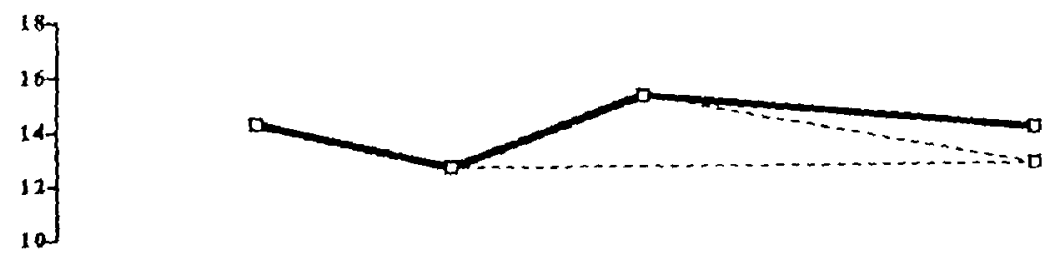

$(-\cos )$

withoul CyA

with CyA

\section{$\underset{\mathrm{mi} / \mathrm{min} / 1.73 \mathrm{m2}}{80}$}

Before BNT

$6 m$
$12 m$

$24 m$

Fig. 1. Plasma uric acid (P.UA), fractional clearance of uric acid (ICUA) and GFR over time in bone-marrow transplant patients on and off CsA (see details in Tables $1,2 a, 2 b$ ).

the bone-marrow transplant patients (same patient before transplantation and after 6 months on CsA; $n=11$ ) and the living related kidney donors/renal transplant patients ('sibling' kidney with and without CsA; $n=23$ ): fractional clearance of uric acid before bone-marrow transplantation $13.0 \% \pm 1.6$ vs $13.3 \% \pm 0.9$ on $\mathrm{CsA}$; fractional clearance of uric acid $10.9 \% \pm 0.8$ in living related kidney donors 1 year after nephrectomy and $10.3 \pm 0.7 \%$ in renal transplant patients 1 year after transplantation.

\section{Discussion}

Hyperuricaemia has been described as a complication of CsA therapy in $30-84 \%$ of patients, depending on 
Table 2a. Fractional clearance of uric acid (fCUA)

\begin{tabular}{|c|c|c|c|c|}
\hline & \multicolumn{4}{|c|}{ fCUA } \\
\hline & $n$ & CsA yes & $n$ & CsA no \\
\hline BMT 0 & & & 27 & $14.3 \pm 1.2^{0 / 1 / 2}$ \\
\hline BMT $6 \mathrm{~m}$ & 21 & $12.7 \pm 0.8^{0 / 1}$ & & \\
\hline $\mathrm{BMT} 12 \mathrm{~m}$ & 13 & $15.3 \pm 2.3^{0 / 1}$ & 13 & $12.8 \pm 1.2^{\circ}$ \\
\hline BMT $18 \mathrm{~m}$ & 11 & $13.2 \pm 1.6^{0 / 1}$ & 11 & $11.7 \pm 0.5^{\circ}$ \\
\hline BMT $24 \mathrm{~m}$ & 7 & $14.1 \pm 1.7^{0 / 1}$ & 16 & $12.8 \pm 0.8^{\circ}$ \\
\hline LRKD 0 & & & 28 & $10.7 \pm 0.1^{1 / 2}$ \\
\hline LRKD ly & & & 28 & $11.1 \pm 0.8^{1 / 3}$ \\
\hline RTP ly & 32 & $11.9 \pm 0.9^{0 / 1}$ & & \\
\hline
\end{tabular}

Data are given as mean $\pm S E M$.

BMT, Bone-marrow transplant patients, $0=$ before, $6,12,18,24$ months after transplantation.

LRKD, Living related kıdney donors, $0=$ before, $1 y=1$ year after unilateral nephrectomy.

RTP Renal transplant patients, $1 \mathrm{y}=1$ year after transplantation

${ }^{0}$ BMT CsA no vs BMT CsA yes/RTP CyA yes, $P$ NS.

${ }^{1} \mathrm{BMT}$ CsA yes/RTP CsA yes vs LRKD $0 / 1 \mathrm{y}, P$ NS.

${ }^{2}$ BMT 0 vs LRKD $0, P<0.05$.

${ }^{3}$ BMT 0 vs LRKD 1y, $P<0.05$.

Table 2b. Inulin clearance $/ 1.73 \mathrm{~m}^{2}$ (CIN 1.73)

\begin{tabular}{lllll}
\hline & \multicolumn{2}{l}{ CIN 1.73 } & & \\
\cline { 2 - 5 } & $n$ & CsA yes & $n$ & CsA no \\
\hline BMT 0 & & & 27 & $106 \pm 6^{0}$ \\
BMT 6m & 21 & $57 \pm 4^{0 / 3}$ & & $79 \pm 7^{1}$ \\
BMT 12m & 13 & $51 \pm 6^{0 / 1 / 2 / 3}$ & 13 & $83 \pm 3^{1}$ \\
BMT 18m & 11 & $56 \pm 7^{0 / 1 / 3}$ & 11 & $79 \pm 5^{1}$ \\
BMT 24m & 7 & $45 \pm 7^{0 / 1 / 3}$ & 16 & $97 \pm 4$ \\
LRKD 0 & & & 28 & $61 \pm 2^{2}$ \\
LRKD 1y & 32 & $49 \pm 3^{2 / 3}$ & 28 & \\
RTP 1y & 32 & & \\
\hline
\end{tabular}

Data are given as mean $\pm S E M$.

BMT, Bone-marrow transplant patients, $0=$ before, $6,12,18,24$ months after transplantation.

LRKD, Living related kidney donors,

$0=$ before, $1 \mathrm{y}=1$ year after unilateral nephrectomy.

RTP, Renal transplant patients, $1 \mathrm{y}=1$ year after transplantation.

${ }^{0}$ BMT 0 vs BMT $6 \mathrm{~m} / 12 \mathrm{~m} / 18 \mathrm{~m} / 24 \mathrm{~m}$ CsA yes, $P<0.001$.

${ }^{1} \mathrm{BMT} 12 \mathrm{~m} / 18 \mathrm{~m} / 24 \mathrm{~m}$ CsA yes vs BMT $12 \mathrm{~m} / 18 \mathrm{~m} / 24 \mathrm{~m}$ CsA no, $P<0.01$.

${ }^{2}$ LRKD $1 \mathrm{y}$ vs BMT $12 \mathrm{~m}$ CsA yes/RTP ly CsA yes, $P<0.05$.

${ }^{3}$ BMT $6 \mathrm{~m} / 12 \mathrm{~m} / 18 \mathrm{~m} / 24 \mathrm{~m}$ CsA yes vs RTP CsA yes, $P$ NS.

how hyperuricaemia is defined [2-4, 9-15]; clinical gout has been reported in $4-24 \%$ of all CsA-treated patients, whereas it is hardly ever noticed in patients under azathioprine [2,4,13-16]. Several authors have speculated about the pathogenesis of this phenomenon. All of them agree that an important cause is the decrease of renal function in CsA-treated patients. Another important factor is the use of diuretics $[2,4,16]$. The turnover rate of uric acid, however, was shown to be not different in CsA-treated patients and in patients receiving azathioprine [2]. Some authors suggest that there is reversible $[5,8,16]$ or irreversible [4] tubular damage, leading to impaired tubular handling of uric acid and resulting in lower fractional clearance of uric acid in CsA-treated patients than in patients under azathioprine.

Our study with serial measurements in bone-marrow transplant patients confirms the existence of hyperuricaemia under CsA as well as its reversibility when CsA is withdrawn and GFR is restored (Table 1/ Figure 1). At 6 months there was a good correlation between plasma uric acid and plasma creatinine $(r=$ $0.75 ; P<0.001)$.

If hyperuricaemia in CsA treated patients were due to tubular dysfunction, resulting in diminished net secretion of uric acid, the fractional clearance of uric acid would be expected to decrease. Serially measured values of the fractional clearance of uric acid of bonemarrow transplant patients, however, did not change significantly over time, regardless of whether patients were on or off CsA (Table 2a/Figure 1). If anything, there was a tendency towards higher values during CsA therapy compared to those after discontinuing it. When intraindividual changes in the fractional clearance of uric acid with vs without CsA were analysed, there were no statistical differences either.

Since it could be conceived that the renal handling of uric acid differs between patients with and without kidney disease (or between patients with one solitary graft-kidney and patients with two normal kidneys), we compared the renal transplant recipients to the bone-marrow transplant patients: their values of fractional clearance of uric acid, however, did not differ. GFR was comparable in both CsA-treated groups (Table $2 a, b$ ). The fractional clearance of uric acid increases only minimally as long as GFR is above $40 \mathrm{ml} / \mathrm{min}$ but increases substantially when GFR falls below $40 \mathrm{ml} / \mathrm{min}$ [17] (see Steele and Rieselbach's controls in Figures 2 and 3). The GFR of the living related kidney donors before and at 1 year after unilateral nephrectomy was higher $(P<0.05)$ than that of the bone-marrow transplant patients and the renal transplant patients receiving CsA. As one would expect from their higher GFR, their fractional clearance of uric acid was somewhat lower, albeit without statistically significant difference (Table $2 a$ ). The fact, that the fractional clearance of the bone-marrow transplant patients before transplantation was significantly higher than that of the living related kidney donors $(P<0.05)$ most probably results from their better hydration ( $3000 \mathrm{ml}$ of i.v. fluid/24 h) and/or drug interference (multidrug regimens before transplantation).

To ensure that the fractional clearance of uric acid of subjects with a healthy, single kidney is comparable to that of subjects with two moderately diseased, but not immunosuppressed kidneys and identical GFR, we compared the results of the fractional clearance of uric acid of our living related kidney donors with those obtained in a group of patients with chronically diseased kidneys of variable degree and origin described by Steele and Rieselbach in 1967 [17] and found no difference. One can easily see in Figure 2 that there is an almost perfect agreement of fractional clearance of uric acid in relation to GFR between Steele's and our patients. When the results of the fractional clearance 


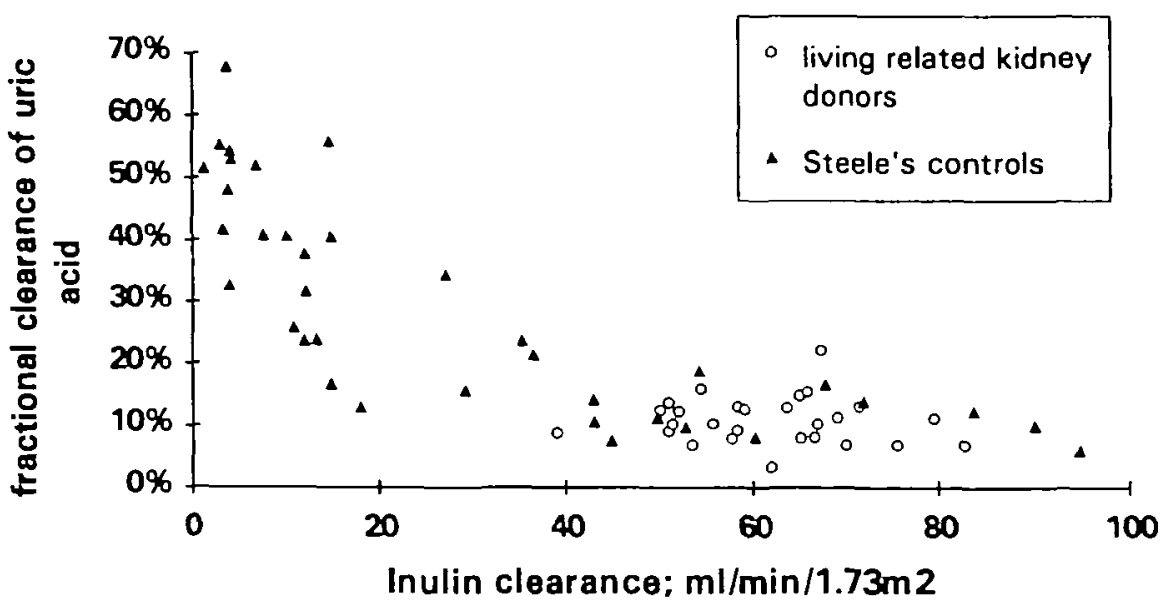

Fig. 2. Fractional clearance of uric acid in relation to inulin clearance in living related kidney donors and in patients with variable degrees of renal failure described by Steele and Rieselbach [17].

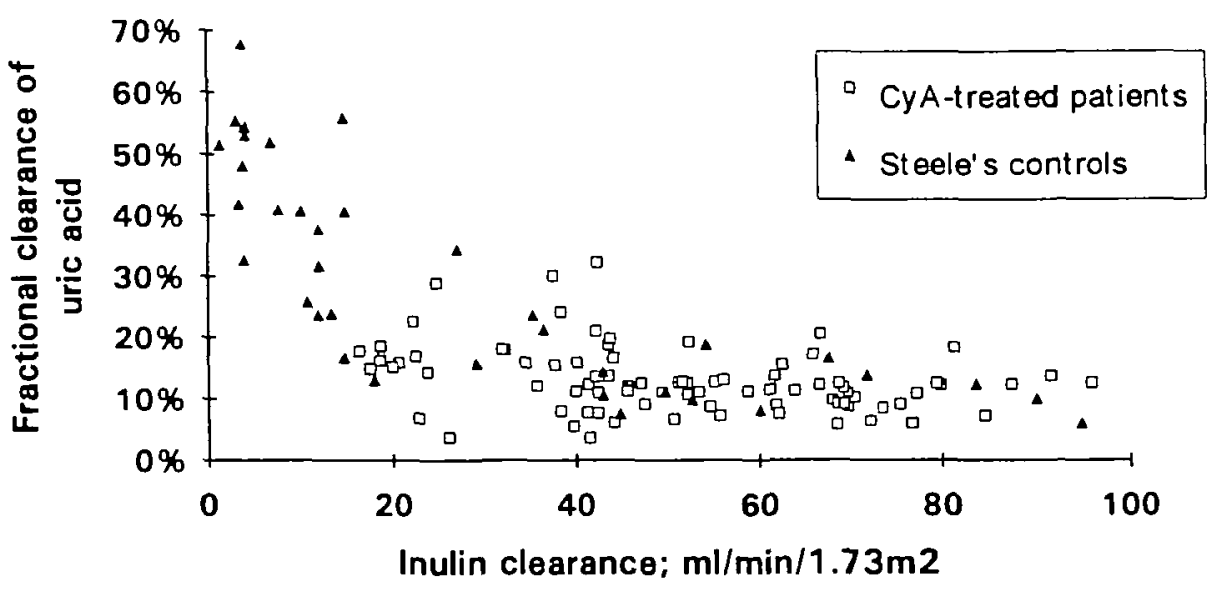

Fig. 3. Fractional clearance of uric acid in relation to inulin clearance in CsA-treated patients and in patients with variable degrees of renal failure described by Steele and Rieselbach [17]

of uric acid of our CsA-treated patients (bone-marrowand renal transplant patients) were graphically superposed on Steele's results, there was again a very good agreement (Figure 3). The latter further confirms our finding that the fractional clearance of uric acid in CsA-treated patients is not different from the fractional clearance of uric acid in subjects with comparable renal function but without CsA.

All reports in the literature describing lower fractional clearance of uric acid in CsA-treated patients as compared to patients on azathioprine refer to creatinine clearance [3-6]. We therefore also calculated the fractional clearances of uric acid in relation to creatinine clearance. The results (data not shown) were exactly congruent in that there were no differences in the fractional clearance of uric acid between groups. Of note, our creatinine-based fractional clearances or uric acid are in the range of $7.3-9.5 \%$, which is in good agreement with those of other authors $[3,13,18]$.

In conclusion, there is no difference in the fractional clearance of uric acid between patients receiving CsA and controls without any immunosuppressive drug. The fractional clearance of uric acid remains unchanged before, during, and after CsA therapy Therefore there is no evidence for impaired tubular handling of uric acid, induced by a CsA-specific tubulotoxic effect. Hyperuricaemia in CsA-treated patients is simply a consequence of CsA-induced diminished renal function and is reversible after its withdrawal, if GFR is restored.

Acknowledgements. This study was supported by clinical study grants of Sandoz-Wander Pharma AG, Roche Pharma AG, and Wellcome AG, Switzerland. We thank Dr A. Scholer (head Clinical-Chemical Laboratories in the Kantonsspital Basel) for analysis of uric acid Furthermore we are indebted to Christa Nolte and Mila Betschart for excellent performance of inulin clearance.

\section{References}

1. European Multicenter Trial Group. Cyclosporine in cadaveric renal transplantation: one-year follow-up of a multicenter trial Lancet 1983; 986-989

2. Lin HY, Rocher LL, McQuillan MA, Schmaltz S, Palella TD, Fox IH. Cyclosporine-induced hyperuricaemia and gout. $N$ Engl J Med 1989; 321: 287-292

3. Noordzij TC, Leunissen KML, van Hooff JP. Letter. $N$ Engl $J$ Med 1990; 322: 335 